\title{
Determining the Most Appropriate Assistive Walking Device Using the Inertial Measurement Unit-Based Gait Analysis System in Disabled Patients
}

\author{
Junhee Lee, $\mathrm{MD}^{1}$, Chang Hoon Bae, $\mathrm{MD}^{1}$, Aeri Jang, $\mathrm{MD}^{1}$, Seoyon Yang, $\mathrm{MD}, \mathrm{PhD}^{2}$, Hasuk Bae, $\mathrm{MD}, \mathrm{PhD}^{1,3}$
}

${ }^{1}$ Department of Rehabilitation Medicine, Ewha Womans University Mokdong Hospital, Seoul;

${ }^{2}$ Department of Rehabilitation Medicine, Ewha Womans University Seoul Hospital, Seoul;

${ }^{3}$ Department of Rehabilitation Medicine, Ewha Womans University College of Medicine, Seoul, Korea

Objective To evaluate the gait pattern of patients with gait disturbances without consideration of defilades due to assistive devices. This study focuses on gait analysis using the inertial measurement unit (IMU) system, which can also be used to determine the most appropriate assistive device for patients with gait disturbances.

Methods Records of 18 disabled patients who visited the Department of Rehabilitation from May 2018 to June 2018 were selected. Patients' gait patterns were analyzed using the IMU system with different assistive devices to determine the most appropriate device depending on the patient's condition. Evaluation was performed using two or more devices, and the appropriate device was selected by comparing the 14 parameters of gait evaluation. The device showing measurements nearer or the nearest to the normative value was selected for rehabilitation. Results The result of the gait evaluation in all 18 patients was analyzed using the IMU system. According to the records, the patients were evaluated using various assistive devices without consideration of defilades. Moreover, this gait analysis was effective in determining the most appropriate device for each patient. Increased gait cycle time and swing phase and decreased stance phase were observed in devices requiring significant assistance.

Conclusion The IMU-based gait analysis system is beneficial in evaluating gait in clinical fields. Specifically, it is useful in evaluating patients with gait disturbances who require assistive devices. Furthermore, it allows the establishment of an evidence-based decision for the most appropriate assistive walking devices for patients with gait disturbances.

Keywords Gait analysis, Assistive device, Rehabilitation, Wearable electronic device, Inertial measurement unit

Received June 12, 2019; Accepted August 22, 2019

Corresponding author: Hasuk Bae

Department of Rehabilitation Medicine, Ewha Womans University Mokdong Hospital, 1071 Anyangcheon-ro, Yangcheon-gu, Seoul 07985, Korea. Tel: +82-2-2650-5035, Fax: +82-2-2650-5145, E-mail: acebhs@ewha.ac.kr

ORCID: Junhee Lee (https://orcid.org/0000-0001-7722-9979); Chang Hoon Bae (https://orcid.org/0000-0003-0677-4261); Aeri Jang (https://orcid. org/0000-0003-2156-8255); Seoyon Yang (https://orcid.org/0000-0002-7522-1655); Hasuk Bae (http://orcid.org/0000-0003-0508-3696).

(c) This is an open-access article distributed under the terms of the Creative Commons Attribution Non-Commercial License (http://creativecommons.org/ licenses/by-nc/4.0) which permits unrestricted noncommercial use, distribution, and reproduction in any medium, provided the original work is properly cited. Copyright $\odot 2020$ by Korean Academy of Rehabilitation Medicine 


\section{INTRODUCTION}

Gait analysis is the systematic study of human motion using various instruments; it measures and analyzes several gait-related parameters such as movement patterns, strength, and joint angle, and range of motion (ROM) [1].

Functional mobility is important because it allows humans to safely perform the activities of daily living, such as walking, running, and climbing. It also helps in handling assistive devices, such as walkers, crutches, and canes [2]. However, approximately $15 \%$ of the world's population live with disability, of which $2 \%-4 \%$ experience significant functional problems [3]. Therefore, the assessment of functional activities is crucial to understand motion disturbances and to determine appropriate management.

The most popular and widespread method that evaluates one's functional mobility is a marker-based motion capture technology using infrared camera. However, this system has some limitations in terms of data recruitment in specific settings and environments. The system requires the usage of an array of camera; therefore, the marker-based motion capture is not applicable to patients requiring assistive devices; the assistive devices are considered as obstacles that may decrease the accuracy of the captured motion. Additionally, its usage is restricted to a specific environment; the system cannot be utilized in the real environments of daily activities, such as in a patient's home or a public field. One potential way to solve this limitation is to use a marker-less motion capture system, the three-dimensional (3D) inertial measurement unit (IMU).

According to the previous studies, sensor technology is one of the emerging methods that can be effectively used in gait analysis. One example of these is an accelerometer [4]. Recently, a simple and cost-effective analysis combined with sensor technology has been used [5]. The inertial sensors generally consist of a gyroscope, an accelerometer, and a magnetometer, allowing the measurements of gravitational force and acceleration [6-12]. During the gait evaluation, changes in the Euler angle, yaw, pitch, and angle of the rolling axis can be measured using the gyroscope [13].

IMUs, referred to as wearable inertial sensors, are widely utilized to evaluate gait patterns in both healthy individuals and patients with gait disturbances. Compared to other types of sensors assessing gait, such as video motion analysis or mat, 3D IMUs are smaller and lighter and are more convenient to be used in the gait analysis of disabled patients who cannot walk without assistance [14]. Additionally, gait evaluation using 3D IMUs allows hourlong ambulatory measurements and evaluation in pointof-care environments [15]. The introduction of IMU also enables the quantitative gait pattern analysis of patients using assistive devices, since the new system does not restrict patients using assistive devices. Additionally, in IMU, the range of study environments is expanded; the gait analysis on the slope or outdoor is available due to its marker-less system.

Throughout the rehabilitation process, it is crucial to identify the most appropriate assistive device for each patient with gait disturbance to reduce the risk of falling and maximize the effect of the rehabilitation. To evaluate the gait pattern of patients with gait disturbances without consideration of defilades due to assistive devices, this study focuses on gait analysis using IMU. In clinical fields, gait disturbance is possibly caused by several diseases. Before starting an individual's rehabilitation, the individual's gait must be evaluated to analyze his/her gait pattern. However, if an individual requires an assistive device, the result of the analysis may be affected by defilades using conventional methods of gait analysis. To solve this problem, gait analysis based on inertial measurement is considered effective due to its marker-less motion capture system. Furthermore, it can also be used to determine the most appropriate assistive device for patients with gait disturbances.

IMU is capable of capturing the patient's gait pattern in quantitative data in various settings and environments; thus, it is considered an effective clinical tool to evaluate gait in patients requiring an assistive device. In this study, we enumerated the advantages of the IMU technologybased gait analysis in clinical fields and the usefulness of this system in determining the most appropriate assistive device for rehabilitation; we utilized the IMU-based gait analysis as a decision-making tool in selecting the most appropriate assistive device for each patient. We reviewed the records and compared the results of the gait analyses based on inertial measurement to confirm its effectiveness in clinical fields and to verify its availability in selecting the most appropriate assistive device for patients requiring an assistive device because of gait distur- 
bance before the initiation of the rehabilitation program.

\section{MATERIALS AND METHODS}

\section{Study design and data source}

This was a retrospective study that used clinical data from the electronic medical records of Ewha Womans University Mokdong Hospital. The study was approved by the Institutional Review Board of Ewha Womans University Mokdong Hospital (No. 2019-03-011). All medical records were fully anonymized before starting the analysis, and all the patients provided informed consent because all data were retrospectively reviewed using the patients' medical records.

\section{Selection of study population}

In this study, we analyzed the data of 18 patients to evaluate their gait using the IMU sensor between May 2018 and July 2018. Both inpatients and outpatients who visited the Department of Rehabilitation Medicine were included in the study. These patients were recently diagnosed with some diseases resulting in gait disturbance. Therefore, these patients required acute phase gait rehabilitation, and an appropriate assistive device before starting the rehabilitation was necessary in these patients.
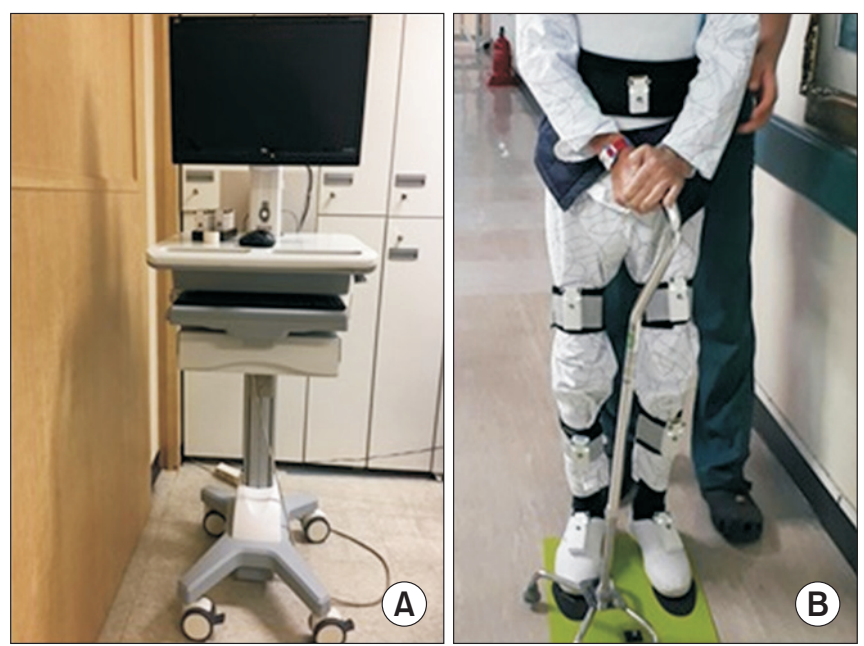

Fig. 1. (A) Gait analysis with inertial measurement unit technology, Human Track (RBioteck Co. Ltd., Seoul, Korea). (B) The IMU sensors are attached to patient's abdomen, bilateral thighs, shanks, and dorsum of both feet.

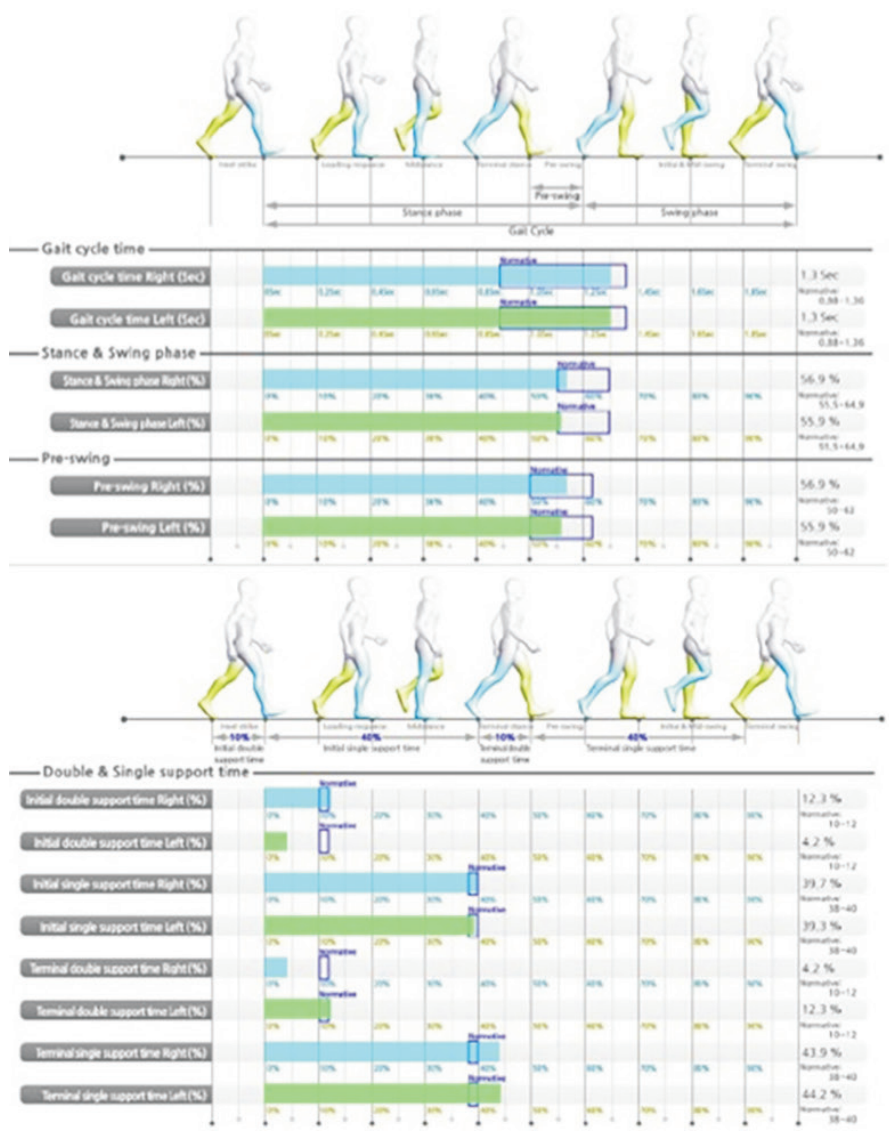

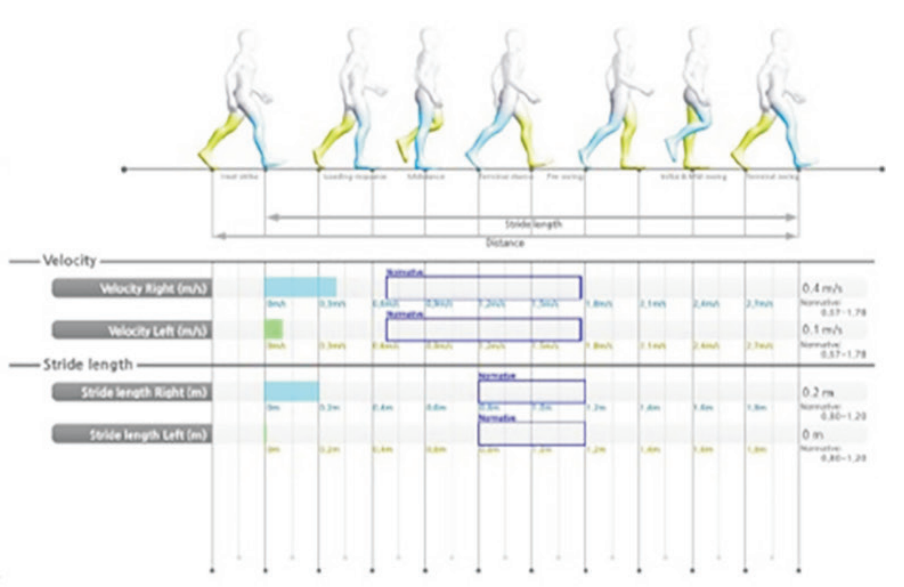

Fig. 2. Example of gait analysis report: gait parameters. 


\section{Characteristics}

According to the data, the instrument used for the evaluation was equipped with a fusion sensor system composed of wireless IMU sensor and stereo camera (Fig. 1). The IMU ( $35 \mathrm{~mm} \times 45 \mathrm{~mm} \times 17 \mathrm{~mm}$ )-based gait analysis system, Human Track (RBiotech Co. Ltd., Seoul, Korea), consisted of a gyroscope, an accelerometer, and magnetometer sensors. A fusion sensor system using a stereo camera was simultaneously applied with the IMU sensor. The IMU sensors were attached to the patient's abdomen, bilateral thighs, shanks, and dorsum of both feet. Once the sensors were attached, the axis calibration was performed by the system. The patient was asked to walk $6 \mathrm{~m}$ several times under the video monitoring, each session with a different assistive device. The system was composed of the following 14 gait parameters: gait cycle
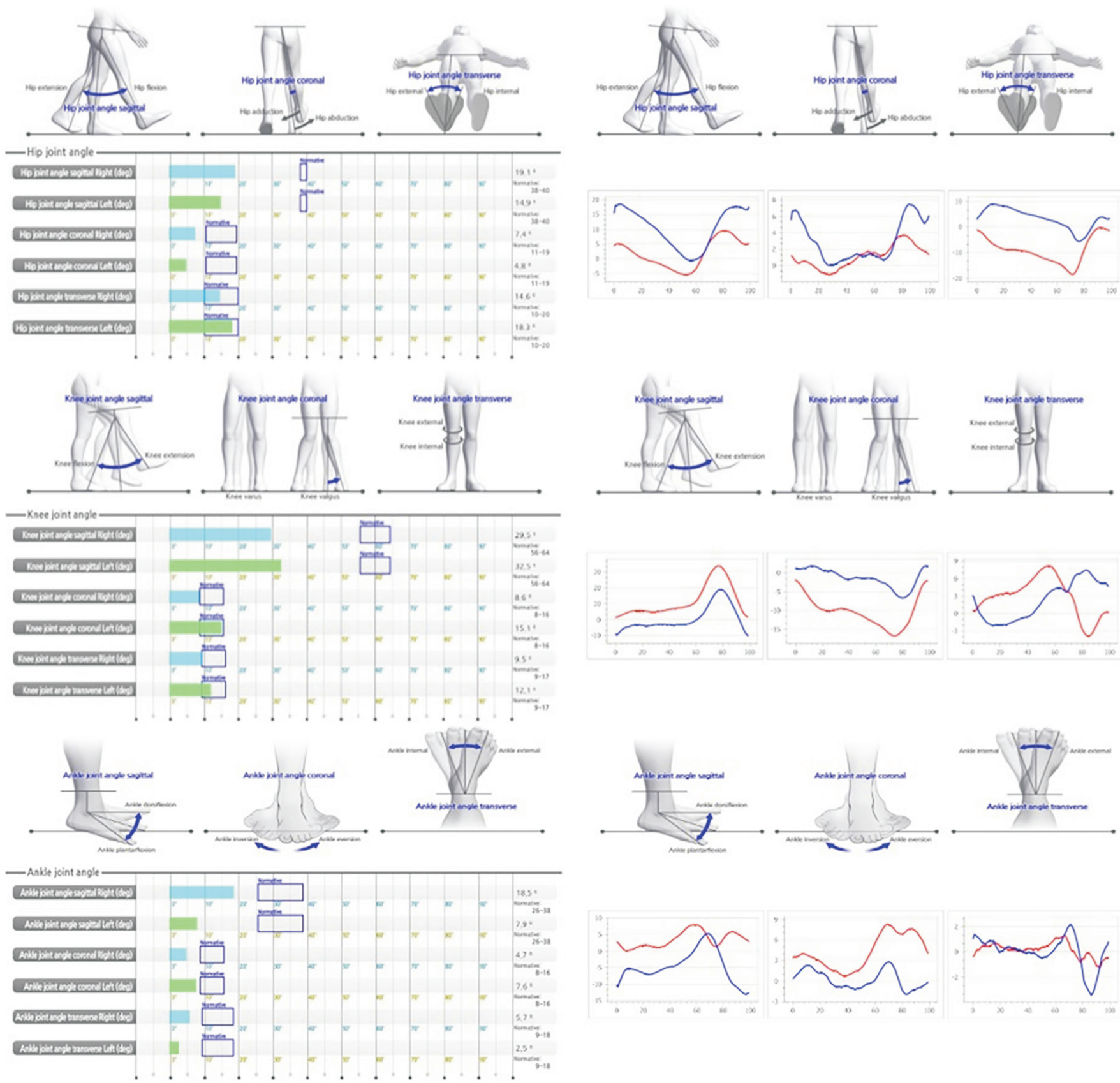

Fig. 3. Example of gait analysis report: (top) angle of hip, (middle) knee, and (bottom) ankle joint (sagittal, coronal, transverse). 
time (sec), stance phase (\%), swing phase (\%), velocity $(\mathrm{m} / \mathrm{s})$, stride length $(\mathrm{m})$, cadence (step/min), pre-swing (\%), initial double support time (\%), initial single support time (\%), terminal double support time (\%), terminal single support time (\%), hip joint angle (o), knee joint angle $\left({ }^{\circ}\right)$, and ankle joint angle ( $\left.{ }^{\circ}\right)$. Figs. 2 and 3 present an example of an IMU gait report. The gathered data were compared with the normative values obtained from 2016 voucher work project supervised by the Institute for Information and Communication Technology Planning and Evaluation. According to this record, the normative values of the parameters were obtained from the IMU evaluation of 200 healthy individuals in their 40 s or older.

\section{Statistical analysis}

We used descriptive statistics to summarize the gait parameters of each assistive device used for the evaluation. The quantitative variables were expressed using median with interquartile range. All statistical analyses were performed using the Statistical Package for the Social Sciences (SPSS) version 25.0 for Windows (IBM SPSS, Armonk, NY, USA).

\section{RESULTS}

The data of the 18 patients ( 12 men, 6 women) were analyzed. Patients' baseline characteristics such as age and sex, underlying diseases, and final choice of assistive device are presented in Table 1. The age range was 11-85 years, and the patients presented with various disease entities including stroke, spinal cord- or spinal rootrelated disease, vertebral fracture, and musculoskeletal problems.

All 18 patients were capable of walking $6 \mathrm{~m}$ using the IMU equipment. The purpose of the evaluation was to select the appropriate device that could realize the patients' gait performance that most closely resembled a normal gait pattern before starting their gait rehabilitation. The result was obtained using two or more devices, and an appropriate device was selected by comparing the 14 parameters as a result of the gait evaluation. Above all, gait velocity was the first priority. The device showing measurement nearer or the nearest to the normative value was selected for rehabilitation. Additionally, if there was no clear difference in the measurements on trials using the devices, the next parameters that we considered were cadence and stride length. The cadence of the nine patients was recorded, and the stride length of the remaining patients was recorded. As discussed previously, if a significant difference was observed during comparison, that is, a device that significantly supported gait had a value closer to that of the normative range, then an inferior-level assistive device, which means a device requiring significant assistance, was used for gait rehabilitation. On the contrary, if a clear difference was not observed, a superior-level handling device, which provides less support, was selected. Furthermore, the percentage of stance and swing phase (ratio) was additionally considered when establishing a decision for the appropriate assistive device. The flowchart of decision-making for the appropriate assistive device comparing the result of the gait evaluation using two or more devices is presented in Fig. 4.

The final choice of assistive device according to the disease entity is summarized in Table 2; 11 patients were able to walk independently, 3 patients used a monocane, one patient preferred to walk using a quad cane, and the last one patient preferred to walk using walker or high walker on their rehabilitation session. As a result, the most preferable choice was gait without assistive devices in patients with stroke and spinal cord- and spinal root-related disease. According to the gait evaluation, the significant differences in gait parameters between the independent gait and the gait using assistive devices were not observed in this study.

The median and interquartile range of each gait parameter were obtained in accordance with the four kinds of assistive devices in this study; the parameters are presented in Table 3. The increase in gait cycle time and swing phase and the decrease in stance phase were observed in inferior-level assistive devices. The gait parameter characteristics of quad-cane were excluded, since only one patient decided to use quad-cane in the rehabilitation program.

\section{DISCUSSION}

Recently, human gait analysis is one of the main subjects of many studies in several clinical fields. Gait pattern is usually associated with locomotion, which can be affected by various diseases. A reliable understanding regarding the several characteristics of gait enables the 
Table 1. Baseline characteristics and determined assistive device

\begin{tabular}{|c|c|c|c|c|}
\hline $\begin{array}{l}\text { Patient } \\
\text { no. }\end{array}$ & $\begin{array}{l}\text { Sex/Age } \\
\text { (yr) }\end{array}$ & Disability & Disease & Assistive device \\
\hline 1 & $\mathrm{M} / 65$ & Right hemiplegia & Left basal ganglia and left frontal ICH & $\begin{array}{l}\text { Mono-cane vs. Quad-cane } \\
\rightarrow \text { Mono-cane }\end{array}$ \\
\hline 2 & $\mathrm{M} / 67$ & Gait disturbance & SAH & $\begin{array}{l}\text { Independent vs. Mono-cane } \\
\rightarrow \text { Independent }\end{array}$ \\
\hline 3 & $\mathrm{~F} / 79$ & Tetraplegia & SCI, AIS D, NLI C4 & $\begin{array}{l}\text { Quad-cane vs. Walker } \\
\rightarrow \text { Quad-cane }\end{array}$ \\
\hline 4 & $\mathrm{M} / 56$ & Gait disturbance & $\begin{array}{l}\text { SDH along the falx and bilateral } \\
\text { tentorium }\end{array}$ & $\begin{array}{l}\text { Independent vs. Mono-cane } \\
\rightarrow \text { Independent }\end{array}$ \\
\hline 5 & $\mathrm{M} / 60$ & Tetraplegia & Cervical myelopathy, C4-5 & $\begin{array}{l}\text { Independent vs. Quad-cane } \\
\rightarrow \text { Independent }\end{array}$ \\
\hline 6 & $\mathrm{M} / 49$ & Right hemiplegia & Left pons ICH & $\begin{array}{l}\text { Independent vs. Quad-cane } \\
\rightarrow \text { Independent }\end{array}$ \\
\hline 7 & $\mathrm{~F} / 76$ & Gait disturbance & Gallbladder stone with cholecystitis & $\begin{array}{l}\text { Mono-cane vs. Walker } \\
\rightarrow \text { Mono-cane }\end{array}$ \\
\hline 8 & $\mathrm{~F} / 60$ & Gait disturbance & Radiculopathy, L3/4 & $\begin{array}{l}\text { Quad-cane vs. Walker } \\
\rightarrow \text { Walker }\end{array}$ \\
\hline 9 & $\mathrm{M} / 85$ & Gait disturbance & $\begin{array}{l}\text { Chronic SDH at left cerebral } \\
\text { convexity, R/O right pons infarction }\end{array}$ & $\begin{array}{l}\text { Compared Mono-cane, } \\
\text { Quad-cane and Walker } \\
\rightarrow \text { walker }\end{array}$ \\
\hline 10 & $\mathrm{M} / 11$ & Gait disturbance & Cauda equina syndrome & $\begin{array}{l}\text { Compared Independent, unilateral } \\
\text { Mono-cane, bilateral Mono-cane } \\
\text { and bilateral Forearm crutch } \\
\rightarrow \text { Independent }\end{array}$ \\
\hline 11 & $\mathrm{~F} / 77$ & Gait disturbance & Compression fracture, $\mathrm{T} 12$ & $\begin{array}{l}\text { Independent vs. Walker } \\
\rightarrow \text { Independent }\end{array}$ \\
\hline 12 & $\mathrm{M} / 60$ & Balance impairment & Left pons infarction & $\begin{array}{l}\text { Independent vs. Mono-cane } \\
\rightarrow \text { Independent }\end{array}$ \\
\hline 13 & $\mathrm{M} / 48$ & Left hemiplegia & Right thalamus ICH & $\begin{array}{l}\text { Independent vs. Mono-cane } \\
\rightarrow \text { Independent }\end{array}$ \\
\hline 14 & $\mathrm{M} / 46$ & Left hemiplegia & Right putamen ICH & $\begin{array}{l}\text { Independent vs. Mono-cane } \\
\rightarrow \text { Independent }\end{array}$ \\
\hline 15 & $\mathrm{~F} / 71$ & Gait disturbance & SAH & $\begin{array}{l}\text { Walker vs. High-walker } \\
\rightarrow \text { High-walker }\end{array}$ \\
\hline 16 & $\mathrm{~F} / 60$ & Gait disturbance & Radiculopathy, L3/4 & $\begin{array}{l}\text { Compared Independent, } \\
\text { Mono-cane and High-walker } \\
\rightarrow \text { Independent }\end{array}$ \\
\hline 17 & $\mathrm{M} / 72$ & Left hemiparesis & Right thalamus ICH & $\begin{array}{l}\text { Independent vs. Mono-cane } \\
\rightarrow \text { Mono-cane }\end{array}$ \\
\hline 18 & $\mathrm{M} / 31$ & Gait disturbance & Degloving injury of left foot & $\begin{array}{l}\text { Independent vs. Left crutch } \\
\rightarrow \text { independent }\end{array}$ \\
\hline
\end{tabular}

ICH, intracerebral hemorrhage; SAH, subarachnoid hemorrhage; SDH, subdural hemorrhage; SCI, spinal cord injury; AIS, American Spinal Injury Association impairment scale grade; NLI, neurological level of injury; C, cervical; L, lumbar.

analysis of abnormal gait pattern caused by diseases and the prediction of the disease prognosis. Moreover, appropriate evaluation at specific time, and monitoring over time, allows the early selection of the most appropriate assistive device in patients with gait disturbances. Therefore, gait analysis is significantly important in clinical 


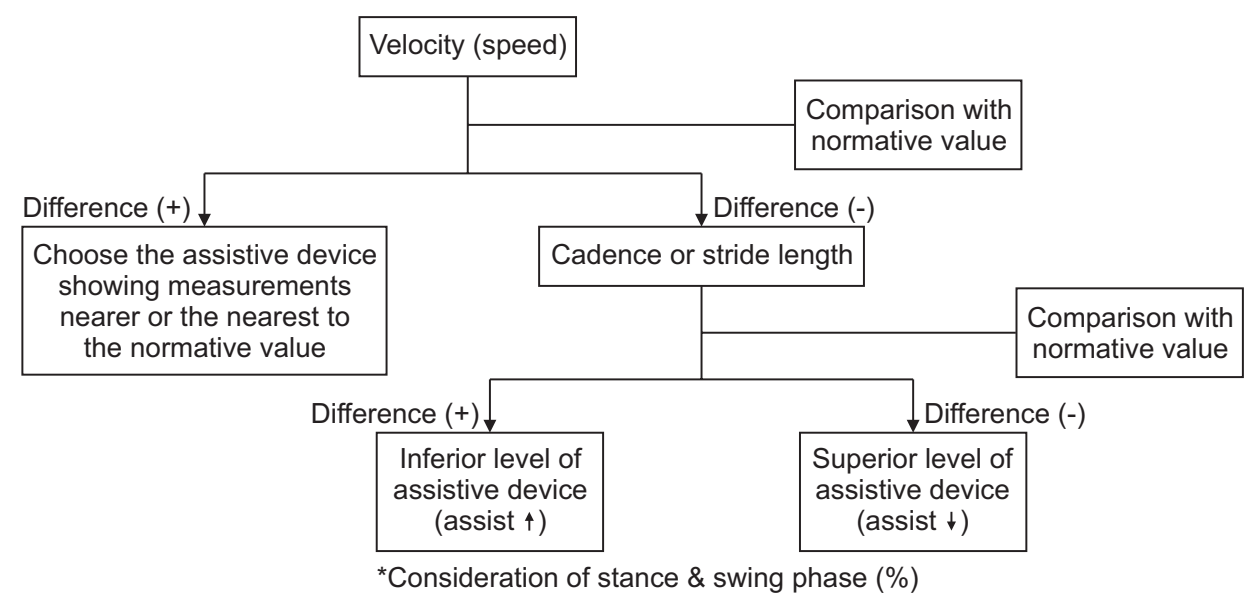

Fig. 4. Flowchart of decision-making of proper assistive device with comparing result of gait evaluation with two or more devices.

Table 2. Choice of device associated with disease category among 18 cases

\begin{tabular}{lcccc}
\hline \multirow{2}{*}{ Disease entity } & \multicolumn{4}{c}{ Assistive device } \\
\cline { 2 - 5 } & Independent & Mono-cane & Quad-cane & Walker or high-walker \\
\hline Stroke & 6 & 2 & - & 2 \\
Spinal cord or root related disease & 3 & - & 1 & 1 \\
Vertebrae fracture & 1 & - & - & - \\
Medical problem & - & 1 & - & - \\
Musculoskeletal problem & 1 & - & - & - \\
\hline
\end{tabular}

Table 3. Descriptive statistics of gait parameters associated with selected device

\begin{tabular}{lcccc}
\hline \multirow{2}{*}{ Gait parameter } & \multicolumn{4}{c}{ Assistive device } \\
\cline { 2 - 5 } & Independent (n=11) & Mono-cane $(\mathbf{n = 3})^{\text {a) }}$ & Quad-cane $(\mathbf{n = 1})$ & Walker or high-walker $(\mathbf{n}=\mathbf{3})^{\mathbf{b})}$ \\
\hline Lt. gait cycle time (s) & $1.40(1.25-1.65)$ & $1.70(1.55-2.00)$ & 1.3 & $1.70(1.70-2.35)$ \\
Rt. gait cycle time (s) & $1.40(1.30-1.60)$ & $1.80(1.60-2.05)$ & 1.2 & $1.70(1.50-1.80)$ \\
Lt. stance phase (\%) & $63.60(58.95-65.05)$ & $58.10(56.80-58.95)$ & 52.3 & $56.90(50.10-60.50)$ \\
Rt. stance phase (\%) & $61.10(58.05-64.30)$ & $56.70(56.15-60.30)$ & 56.7 & $57.80(55.45-60.85)$ \\
Lt. swing phase (\%) & $36.40(34.95-41.05)$ & $41.90(41.05-43.20)$ & 47.7 & $43.10(39.50-49.90)$ \\
Rt. swing phase (\%) & $38.90(35.70-41.95)$ & $43.30(39.70-43.85)$ & 43.3 & $42.20(39.15-44.55)$ \\
Lt. velocity (m/s) & $0.80(0.50-0.95)$ & $0.70(0.60-0.80)$ & 0.7 & $0.60(0.45-0.65)$ \\
Rt. velocity (m/s) & $0.80(0.55-0.95)$ & $0.60(0.55-0.60)$ & 0.7 & $0.60(0.50-0.85)$ \\
Lt. stride length (m) & $0.70(0.40-0.90)$ & 0.5 & 0.5 & $0.30(0.10-0.50)$ \\
Rt. stride length (m) & $0.70(0.40-0.85)$ & 0.8 & 0.5 & $0.35(0.20-0.50)$ \\
Lt. cadence (step/min) & $87.30(73.50-96.85)$ & 87.5 & - & 70.1 \\
Rt. cadence (step/min) & $85.00(75.30-97.15)$ & 88.4 & - & 62.2 \\
\hline
\end{tabular}

Values are presented as median (interquartile range).

${ }^{a)}$ Stride $(n=2)$ and cadence $(n=1),{ }^{\text {b) }}$ Cadence $(n=1)$.

practice because it offers information about the patient's functional capacity and helps in planning effective rehabilitation program individually [16].

There are various types of technological devices that can be used in human gait analysis. These are categorized according to two kinds of methodology. One is a nonwearable sensor (NWS), and another is a wearable sensor (WS). NWS systems require controlled research facilities 
for the sensors' placement and capture data while the subject is walking on a specific pathway. In contrast, WS systems allow the analysis of gait pattern without consideration of the surroundings and capture information while the subject performs the activities of daily living. Specifically, WS systems use sensors located on several parts of the body, such as the feet, knees, thighs, or waist. Different kinds of sensors are included in this category.

The traditionally used methods to evaluate human gait are expensive and complicated. Considering these limitations, these methods are not regularly used in clinical practice. To resolve this problem, inertial sensor-based gait analysis has been used. IMU sensor is composed of accelerometers, gyroscopic sensors, and magnetometers. Additionally, force sensors, extensometers, goniometers, active markers, and electromyography are utilized to capture the signals that characterize the gait [17]. Sant'Anna et al. [18] developed a system with inertial sensors to quantify gait symmetry and gait normality. The evaluation was performed in the laboratory, as opposed to the $3 \mathrm{D}$ kinematic measurements, and in situ, contrary to the clinical assessments of patients undergoing hip replacement. The results showed good correlation factor between the different methods. Furthermore, Ferrari et al. [19] presented an algorithm to measure gait parameters, which were compared with the outcomes of gold standard camera-based system. It presented a difference in step length less than $5 \%$ with consideration of the median values. More recently, Petraglia et al. [20] showed a good agreement between the IMUs and classical gait analysis for several gait parameters; therefore, it finally confirmed that IMUs can be used to capture kinematic information in different environments and time frames and can even be performed outside a laboratory in real-life conditions. The IMU system appears to be an accurate and repeatable measuring tool to evaluate the spatiotemporal gait parameters in healthy adults [21]. Furthermore, it is useful in evaluating the gait patterns of patients with neurological diseases [14,22,23]. Additionally, IMU can measure disease severity [24].

Assistive devices play an important role in supporting gait. However, studies that determine the appropriate assistive device are limited because it is difficult to measure and interpret the gait pattern when devices are used. Therefore, it is necessary to create an algorithm regarding the establishment of a decision for the most appropriate assistive device to further develop machine learning and artificial intelligence (AI) system. According to the previous studies, Rafael et al. summarized the studies that applied adaptive, also called AI, algorithms to gait analysis based on inertial sensor data, validating if they can be used in clinical fields [16]. Additionally, Cho et al. [25] presented scoliosis screening with machine learning using the IMU-based gait analysis. Based on this background, this study focused on developing a basic algorithm suggesting the most appropriate assistive device to patients with gait disturbances using a gait evaluation tool that is not affected by defilades. Additionally, we aimed to confirm this concept in clinical fields.

Eighteen patients with gait abnormality were evaluated using the IMU equipment. All patients were evaluated using various assistive devices to determine the most appropriate device for gait rehabilitation. When the patients were evaluated using conventional gait analysis such as treadmill, patients requiring assistive devices were frequently excluded in the evaluation of gait patterns because the devices were considered as obstacles on the gait evaluation and the risk of falling without the device was high. The IMU-based gait analysis is capable of overcoming such problem, providing quantitative information on the gait parameters to all patients. Furthermore, it is beneficial in determining the most appropriate assistive device for patients by comparing the gait parameters of each device.

Based on the result of our analysis, our study presented two major findings. First, gait using assistive devices in patients with stroke and spinal cord- and spinal rootrelated diseases did not show a statistically significant difference to independent gait. Second, increase in gait cycle time and swing phase and decrease in stance phase were observed in inferior-level assistive devices, which provide more support on gait evaluation.

This study has three limitations. First, the patient's disease entity was heterogeneous. It can be considered as a limitation because it provides possible inconsistencies in the patterns of the gait parameters according to the patient's condition. However, on the contrary, it can be considered as a strength in this study because it shows that IMU-based gait analysis is available without consideration of patient's disease entity and patterns of disability. Second, there the difference between sound side and lesion side was not discussed. According to the disease, the 
affected side and disability pattern can possibly vary, and it may have an effect on gait parameters. Further study comprising a large sample size that can be classified into disease category or disability patterns is required. Third, normative value used in this study was obtained from the IMU evaluation of 200 healthy individuals in their 40 s or older, providing the possibility of generalization error; therefore, creating the control group or developing the detailed criteria about the gait parameters of IMU-based gait analysis can be considered as a possible solution.

Conventional studies that evaluate the gait patterns of the patients using the assistive devices are frequently limited mostly due to their technical limitations. The marker-less IMU-based gait analysis broadens the scope of the gait evaluation by measuring quantitative information of gait patterns regardless of the surrounding conditions or the presence of assistive devices. To discuss future plans in using IMU-based gait analysis on gait correction and selection of appropriate assistive devices, further study that can resolve the limitations mentioned previously is required. Additionally, not only focusing on time-spatial and ROM range but also concentrating on choosing the principal component on the real gait pattern and applying the machine learning method is critical on future studies.

In conclusion, prior to the initiation of rehabilitation, the IMU-based gait analysis is effective in establishing the decision regarding the most appropriate assistive device in patients with gait disturbances. With the quantitative information and analyses on gait parameters using different assistive devices, an evidence-based decision regarding the most appropriate device on one's rehabilitation can be established.

\section{CONFLICT OF INTEREST}

No potential conflict of interest relevant to this article was reported.

\section{AUTHOR CONTRIBUTION}

Conceptualization: Lee JH, Bae HS. Methodology: Lee JH, Jang AR, Bae CH. Formal analysis: Lee JH. Funding acquisition: Bae HS. Project administration: Lee JH, Bae HS. Visualization: Lee JH, Bae HS. Writing - original draft: Lee JH. Writing - review and editing: Lee JH, Bae
$\mathrm{CH}$, Yang SY, Bae HS. Approval of the final manuscript: all authors.

\section{REFERENCES}

1. Sharif Bidabadi S, Murray I, Lee GYF. The application of inertial measurements unit for the clinical evaluation and assessment of gait events. J Med Eng Technol 2017;41:612-22.

2. Vargas-Valencia LS, Elias A, Rocon E, Bastos-Filho T, Frizera A. An IMU-to-body alignment method applied to human gait analysis. Sensors (Basel) 2016;16:E2090.

3. World Health Organization, World Bank. World report on disability. Geneva, Switzerland: World Health Organization; 2011.

4. Lee JH, Park SW, Kim DA, Jang SJ, Kim YH. Gait analysis using accelerometer in stroke patients. J Korean Acad Rehabil Med 2004;28:488-93.

5. Cho YS, Jang SH, Cho JS, Kim MJ, Lee HD, Lee SY, et al. Evaluation of validity and reliability of inertial measurement unit-based gait analysis systems. Ann Rehabil Med 2018;42:872-83.

6. Favre J, Jolles BM, Aissaoui R, Aminian K. Ambulatory measurement of 3D knee joint angle. J Biomech 2008;41:1029-35.

7. Watanabe T, Saito H. Tests of wireless wearable sensor system in joint angle measurement of lower limbs. Conf Proc IEEE Eng Med Biol Soc 2011;2011:5469-72.

8. van Acht V, Bongers E, Lambert N, Verberne R. Miniature wireless inertial sensor for measuring human motions. Conf Proc IEEE Eng Med Biol Soc 2007;2007:6279-82.

9. Luinge HJ, Veltink PH. Measuring orientation of human body segments using miniature gyroscopes and accelerometers. Med Biol Eng Comput 2005;43:27382.

10. Zhu R, Zhou Z. A real-time articulated human motion tracking using tri-axis inertial/magnetic sensors package. IEEE Trans Neural Syst Rehabil Eng 2004;12:295302.

11. Kim MS, Yu SB, Lee KS. Development of a high-precision calibration method for inertial measurement unit. Int J Precis Eng Manuf 2014;15:567-75.

12. Lee D, Lee S, Park S, Ko S. Test and error parameter estimation for MEMS: based low cost IMU calibration. Int J Precis Eng Manuf 2011;12:597-603. 
13. Madgwick $S$. An efficient orientation filter for inertial and inertial/magnetic sensor arrays [Internet]. Bristol, UK: X-IO Technologies; 2010 [cited 2020 Jan 15]. Available from: http://x-io.co.uk/res/doc/madgwick_ internal_report.pdf.

14. Vienne A, Barrois RP, Buffat S, Ricard D, Vidal PP. Inertial sensors to assess gait quality in patients with neurological disorders: a systematic review of technical and analytical challenges. Front Psychol 2017;8:817.

15. Maetzler W, Domingos J, Srulijes K, Ferreira JJ, Bloem BR. Quantitative wearable sensors for objective assessment of Parkinson's disease. Mov Disord 2013;28:1628-37.

16. Caldas R, Mundt M, Potthast W, Buarque de Lima Neto F, Markert B. A systematic review of gait analysis methods based on inertial sensors and adaptive algorithms. Gait Posture 2017;57:204-10.

17. Muro-de-la-Herran A, Garcia-Zapirain B, MendezZorrilla A. Gait analysis methods: an overview of wearable and non-wearable systems, highlighting clinical applications. Sensors (Basel) 2014;14:3362-94.

18. Sant'Anna A, Wickstrom N, Eklund H, Zugner R, Tranberg R. Assessment of gait symmetry and gait normality using inertial sensors: in-lab and in-situ evaluation. In: Gabriel J, Schier J, Van Huffel S, Conchon E, Correia C, Fred A, Gamboa H, editors. Biomedical engineering systems and technologies. Heidelberg: Springer; 2013. p. 239-54.

19. Ferrari A, Rocchi L, van den Noort J, Harlaar J. Toward the use of wearable inertial sensors to train gait in subjects with movement disorders. In: Pons J, Torricelli D, Pajaro M, editors. Converging clinical and engineering research on neurorehabilitation. Heidelberg: Springer; 2013. p. 937-40.

20. Petraglia F, Scarcella L, Pedrazzi G, Brancato L, Puers $\mathrm{R}$, Costantino C. Inertial sensors versus standard systems in gait analysis: a systematic review and metaanalysis. Eur J Phys Rehabil Med 2019;55:265-80.

21. Washabaugh EP, Kalyanaraman T, Adamczyk PG, Claflin ES, Krishnan C. Validity and repeatability of inertial measurement units for measuring gait parameters. Gait Posture 2017;55:87-93.

22. Brognara L, Palumbo P, Grimm B, Palmerini L. Assessing gait in Parkinson's disease using wearable motion sensors: a systematic review. Diseases 2019;7:E18.

23. Zago M, Sforza C, Pacifici I, Cimolin V, Camerota F, Celletti C, et al. Gait evaluation using inertial measurement units in subjects with Parkinson's disease. J Electromyogr Kinesiol 2018;42:44-8.

24. Vienne-Jumeau A, Quijoux F, Vidal PP, Ricard D. Value of gait analysis for measuring disease severity using inertial sensors in patients with multiple sclerosis: protocol for a systematic review and meta-analysis. Syst Rev 2019;8:15.

25. Cho JS, Cho YS, Moon SB, Kim MJ, Lee HD, Lee SY, et al. Scoliosis screening through a machine learning based gait analysis test. Int J Precis Eng Manuf 2018;19:1861-72. 